International Journal of Advanced Research in Engineering and Technology (IJARET)

Volume 10, Issue 4, July-August 2019, pp. 47-54, Article ID: IJARET_10_04_006

Available online at http://iaeme.com/Home/issue/IJARET?Volume $=10 \&$ Issue $=4$

ISSN Print: 0976-6480 and ISSN Online: 0976-6499

(C) IAEME Publication

\title{
NUMERICAL SIMULATION OF VELOCITY AND PRESSURE FIELD AROUND A CYLINDER AT MODERATE REYNOLDS NUMBER
}

\author{
F. Minniti
}

PHD Student, Department of Civil Engineering, Energy, Environmental and Materials, Mediterranea University, Reggio Calabria, Italy.

\section{Fiamma}

Researcher, Department of Civil Engineering, Energy, Environmental and Materials, Mediterranea University, Reggio Calabria, Italy.

\section{G. Barbaro}

Professor, Department of Civil Engineering, Energy, Environmental and Materials, Mediterranea University, Reggio Calabria, Italy.

\section{P. Puntorieri}

PHD, Department of Civil Engineering, Energy, Environmental and Materials, Mediterranea University, Reggio Calabria, Italy.

\begin{abstract}
Many offshore structures contain cylindrical elements, such as cladding platforms, jackets, tension-leg platforms and marine pipelines. However, exposure to current and waves can cause oil leakage. Conducts at intermediate water depths are subject to currents plus waves causing large variations in velocities and hence the Reynolds number (Re $=U c D / v$, where $U c$ is the free flow velocity, $D$ is the diameter of the cylinder and $v$ is the kinematic viscosity of the fluid) most often appears in the range from $10^{\wedge} 3$ to $10^{\wedge} 7$. It is therefore important to understand the flow around the circular cylinder in the effective Re range.

In recent years there is a strong use of computational fluid dynamics (CFD) which is a branch offluid mechanics that uses numerical analysis and data structures to solve and analyze problems involving fluid flow and also interaction between waves and structures. The main use of CFD is to solve the Navier-Stokes equations and their equations. There are therefore several methods to solve the Navier-Stokes equations, and since they are generally high-cost computational operations, more sophisticated model-based approaches have been developed:
\end{abstract}

- Direct numerical simulation 
- Reynolds Averaged Navier-Stokes (RANS)

- Large Eddy Simulation (LES)

This paper deals with the use of CFD to determine the flow field concerning the complex interaction of a cylindrical structure with a fluid using the approach based on Reynolds Averaged Navier-Stokes (RANS).

Key words: Offshore structures, CFD, RANS, velocity field, pressure field, numerical methods.

Cite this Article: F. Minniti, V. Fiamma, G. Barbaro and P. Puntorieri, Numerical Simulation of Velocity and Pressure Field Around a Cylinder at Moderate Reynolds Number, International Journal of Advanced Research in Engineering and Technology, 10(4), 2019, pp. 47-54.

http://iaeme.com/Home/issue/IJARET?Volume $=10 \&$ Issue $=4$

\section{INTRODUCTION}

Vortex shedding behind two-dimensional circular cylinders has been one of the most studied subjects in fluid mechanics in the past several decades because of its practical and theoretical importance. Although a quite comprehensive understanding of the vortex dynamics in a cylinder's wake has been achieved (as reflected in the reviews by Williamson (1996) and many other researchers), the simplicity of the geometry and the abundance of interesting flow features continue to make this phenomenon the subject of many current studies. During the last years, many studies were performed in order to investigate wave forces on vertical cylinders, for example [1],[2],[3],[4],[5]; pipelines at intermediate water depths are also subjected to currents plus waves causing large variations in the velocities and thereby Reynolds number ( $R e=U_{c} D / v$, where $U_{c}$ is the free stream velocity, $D$ is the diameter of the cylinder and $v$ is the kinematic viscosity of the fluid), most often appearing in the range from $10^{3}$ to $10^{7}$. The numerical research of the flow with comparable $R e$ is limited, while the higher $R e$ cases are studied in several publications.

\section{RANS NUMERICAL MODEL}

The Reynolds averaged Navier-Stokes equations, which are often referred to as RANS, are based on the assumption that turbulent motion can be seen as formed by a mean motion and its fluctuation over time. The magnitudes of starting equations are averaged over a certain period of time; in this way calculation times are considerably reduced as the scales of the mean motion are considerably greater than those of the turbulent motion.

These equations can be used with approximations based on knowledge of the properties of flow turbulence to give approximate time-averaged solutions to the Navier-Stokes equations. For a stationary, incompressible Newtonian fluid, these equations can be written in Einstein notation in Cartesian coordinate as:

$$
\rho \overline{\mathrm{u}}_{\mathrm{j}} \frac{\partial \overline{\mathrm{u}_{1}}}{\partial \mathrm{x}_{\mathrm{j}}}=\rho \overline{\mathrm{f}_{\mathrm{i}}}+\frac{\partial}{\partial \mathrm{x}_{\mathrm{j}}}\left[-\overline{\mathrm{p}} \delta_{\mathrm{ij}}+\mu\left(\frac{\partial \overline{\mathrm{u}_{1}}}{\partial \mathrm{x}_{\mathrm{j}}}+\frac{\partial \overline{\mathrm{u}_{\mathrm{j}}}}{\partial \mathrm{x}_{\mathrm{i}}}\right)-\rho \overline{\overline{\mathrm{u}}_{1}{ }_{1} \mathrm{u}^{\prime}{ }_{\mathrm{j}}}\right]
$$

\section{RANS CALIBRATION}

In the present study a moderate Re value is chosen, corresponding to the standard operating conditions of the marine pipelines which gives a whirling road, but the flow is still not very 
turbulent. The frequency and amplitude of the oscillations are stable characteristics, but the flow details are extremely sensitive to perturbations. It is important to note that this sensitivity is a physical reality and not simply a numerical artifact.

The typical approach requires to discretize the fluid domain in elementary cells so as to obtain a calculation grid (also called mesh), on which to apply iterative resolution methods in order to solve the Navier-Stokes equations.

In the different approaches highlighted the analysis procedure is always, in general terms, similar:

1. The geometry (or physical domain) of the problem to be analyzed is defined.

2. The volume occupied by the fluid is discretized, i.e. divided into a large number of elementary cells, generating a calculation grid.

3. The physical model is defined (for example, the equations of motion + the equation of energy + the equations of the species) and therefore the numerical one (method of discretization of the equations, algorithms for the resolution of the equations).

4. The boundary conditions are defined, ie the properties of the fluid in the calculation domain are specified. For time-dependent problems, the initial conditions must be specified.

5. The equations are solved in an iterative manner. The calculation is interrupted once the desired degree of accuracy has been reached.

6. The results produced are displayed with a post-processor.

7. Finally, the analysis of the results is carried out.

The software used in this study is COMSOL Multiphysics, that is is a powerful interactive modeling environment to solve all kinds of scientific and engineering problems. When solving the models, COMSOL Multiphysics uses the Finite Element Method (FEM); The software performs finite element analysis together with mesh and error checking using a variety of numerical solvers.

\section{NUMERICAL VALIDATION}

The simulation started from the definition of geometry of the problem. An area of $110 \mathrm{~m} \times 35$ $\mathrm{m}$ was chosen, where a cylinder with a diameter of $15 \mathrm{~m}$ was positioned, $10 \mathrm{~m}$ along $\mathrm{x}$-axis and $10 \mathrm{~m}$ along y-axes.

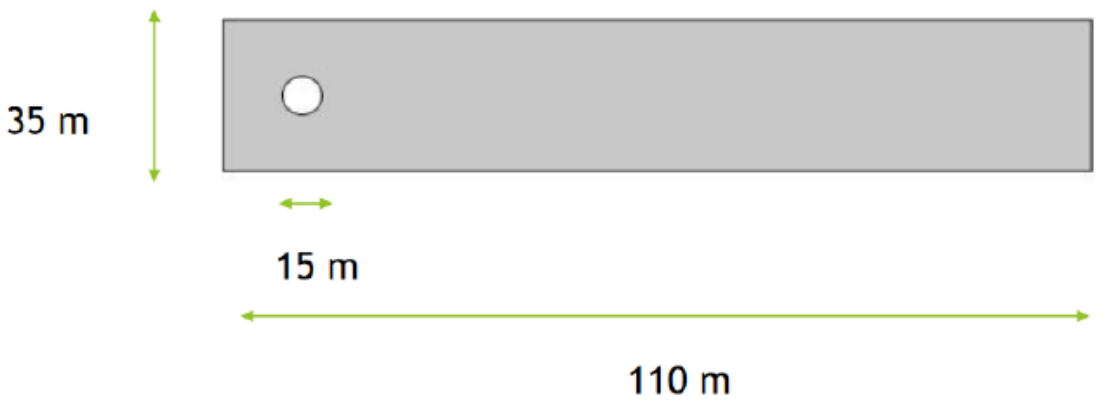

Figure 1 Scheme of the calculation domain

The domain has been discretized through a mesh controlled by the physics of the problem that is denser in correspondence with the walls of the domain and on the solid contour of the 
cylinder. In this case, an unstructured mesh is used, and the small asymmetry in the mesh proves to be enough to trigger the vortex production.

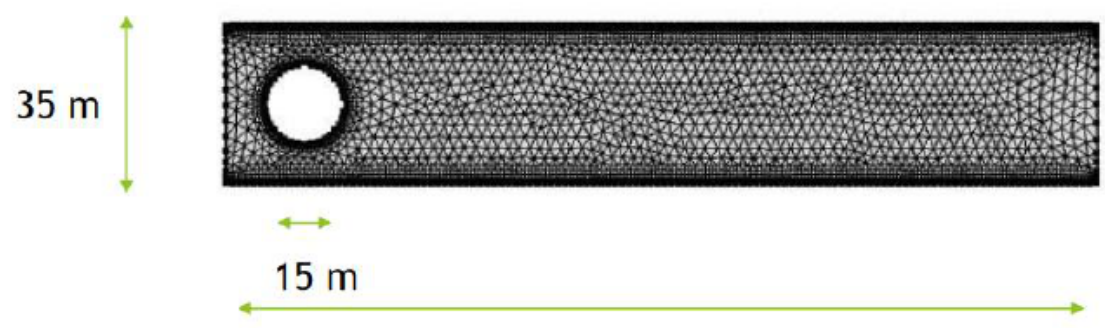

$110 \mathrm{~m}$

Figure 2 Domain discretization

To illustrate how you can study such effects, the following model examines unsteady, incompressible flow past a long cylinder placed in a channel at right angle to the incoming fluid. With a symmetric inlet velocity profile, the flow needs some kind of asymmetry to trigger the vortex production. This can be achieved by placing the cylinder with a small offset from the centre of the flow.

A time dependent study was then set up for the CFD simulation, in order to study velocity and pressure field over time. A range of Reynolds numbers is investigated $\left(\mathrm{Re}=1.10^{4}\right.$ to $\left.5.10^{6}\right)$, encompassing the critical regime and an incompressible, turbulent flow of a Newtonian fluid is considered. Reynolds averaged Navier-Stokes and continuity equations are solved for twodimensional flow.

At the inlet, a unidirectional flow and spatially constant variables are assumed. Inlet boundary conditions for turbulence quantities are derived by assuming a very low turbulence intensity in order to overcome the problems of stability of the solution. The time range of simulation is $(0 ; 1.4 ; 21) \mathrm{s}$, this time range seemed suitable for the observation of the phenomenon on the whole domain, here are the velocity field for some time steps:

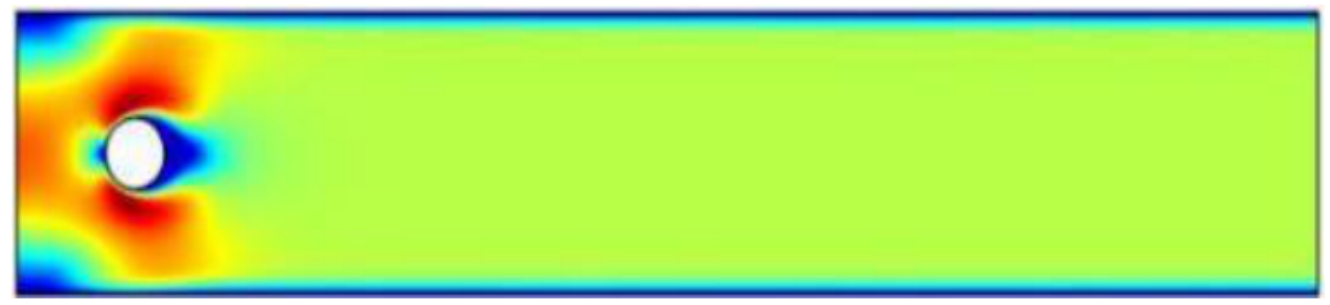

Figure 3 Velocity field at time step $1.4 \mathrm{~s}$

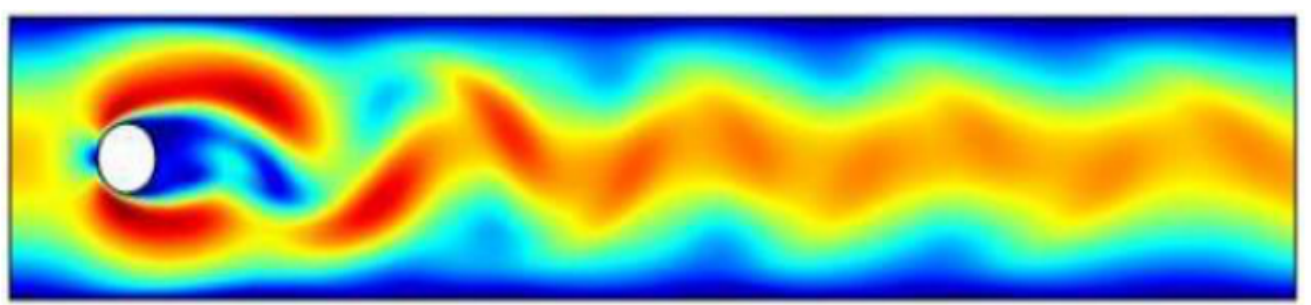

Figure 4 Velocity field at time step $18.2 \mathrm{~s}$ 
The software has simulated the field of motion and pressure within the computational domain; it is showed how in the initial phase there is a velocity increase in the area of the cylinder in front of the wall from which the flow field is generated; with the passing of the time a turbulent flow is generated around the structure with an increase in velocity in the shadow zone of the cylinder initially, and in the whole computation domain until the end of the simulation.

Here are the pressure field for some time step:

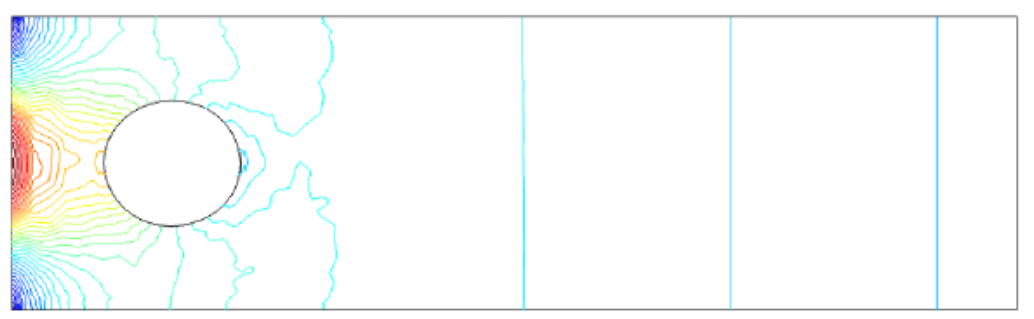

Figure 5 Pressure field at time step $1.4 \mathrm{~s}$

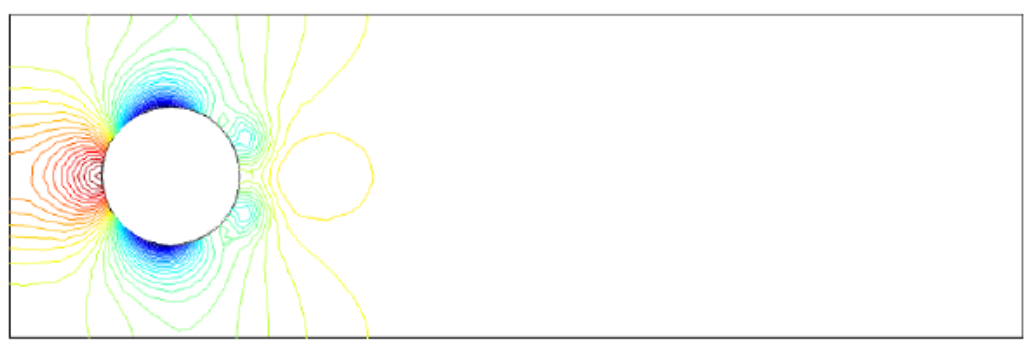

Figure 6 Pressure field at time step $18.2 \mathrm{~s}$

Regarding the pressure field, there is an increase of pressure in the area in front of the wall from which the flow of the field of motion is generated, consistent with the physical model analyzed, and a depression zone in the shadow zone of the cylinder; as time changes, however, pressure value increases throughout the computation domain. This is in according with field experiments by Boccotti $(1995,1996)$, that used wave pressure measurements and QD theory to show how the wave field is modified by the presence of a cylinder structure, in particular the presence of an overpressure at the side of the cylinder exposed to the motion field and a corresponding under pressure at the side protected by the presence of the cylinder itself. It was fixed a time step of $4.2 \mathrm{~s}$ that is significant to show how the pressure field is distributed in front of and behind the cylinder. It was considered an inline plot fixing $y$-axis at $y=17.5 \mathrm{~m}$, that is the center of the cylinder, by varying $\mathrm{x}$-axis considering firstly the area in front of the wall (from $\mathrm{x}=0 \mathrm{~m}$ to $\mathrm{x}=10 \mathrm{~m}$ ) and then the area in the shadow zone of the cylinder (from $\mathrm{x}=25 \mathrm{~m}$ to $\mathrm{x}=35 \mathrm{~m})$.

In the y-axis of the inline plot it was considered the wave pressure normalized with the maximum itself, and in the x-axis the horizontal position normalized with the radius of the cylinder. 


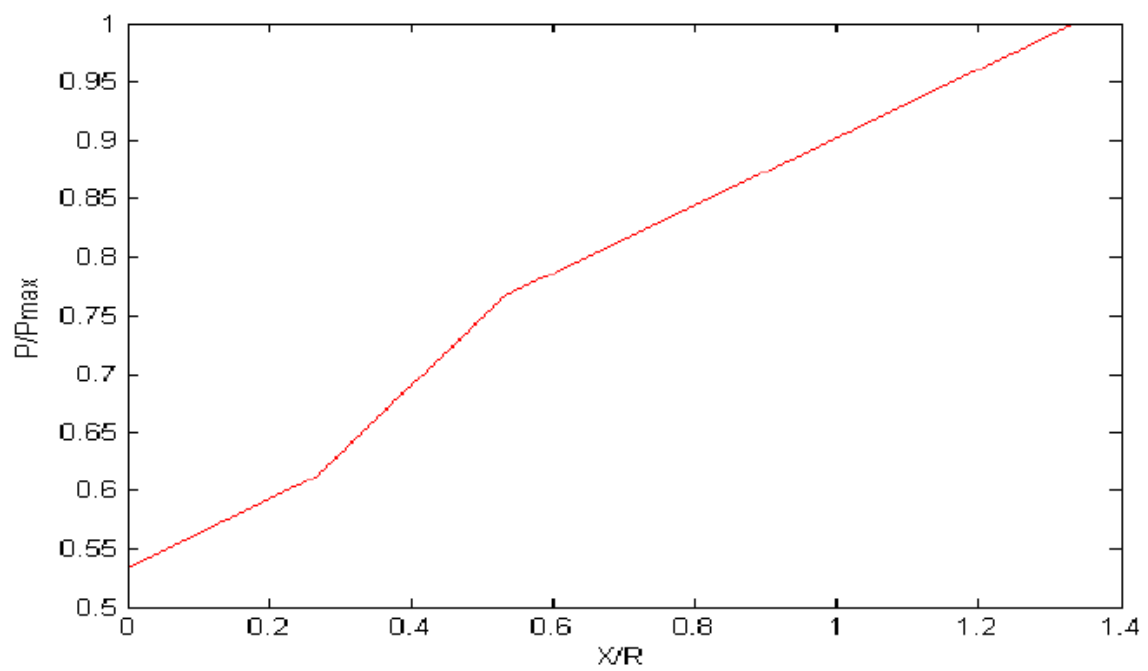

Figure 7 Normalized wave pressure in front of the cylinder at the center $y=17.5 \mathrm{~m}$.

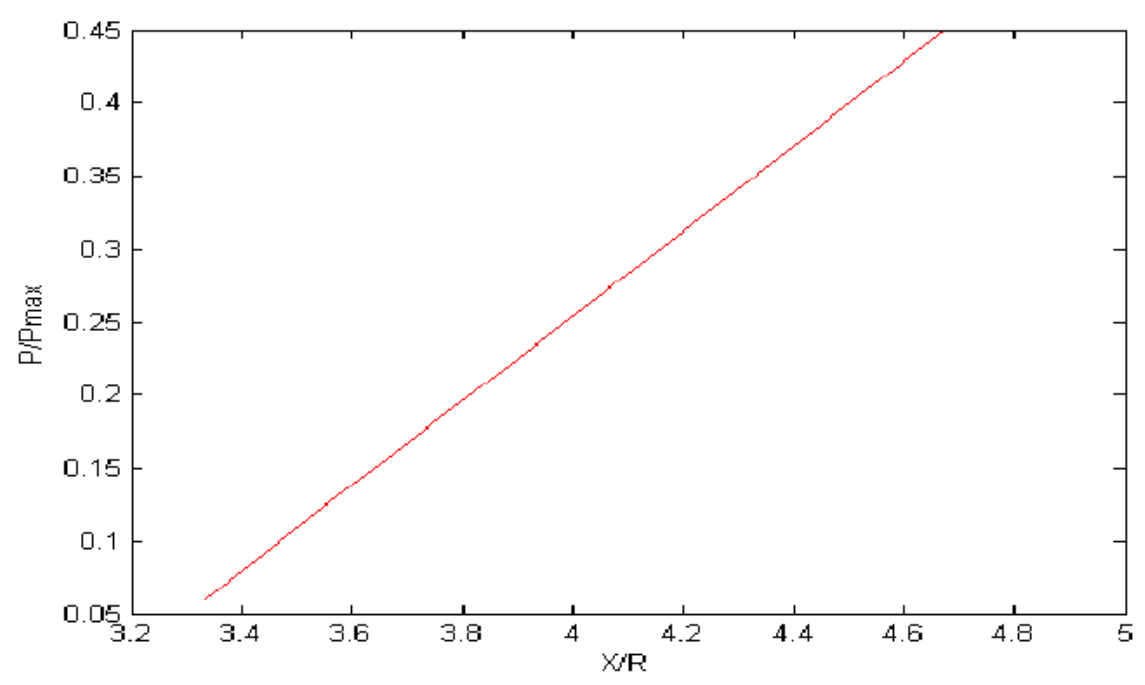

Figure 8 Normalized wave pressure behind the cylinder at the center $y=17.5 \mathrm{~m}$.

It can be noticed an increase in pressure from the position of the source of the flow field which reaches a maximum in front of the cylinder, and a depression area behind the cylinder which increases slightly away from it.

Regarding the drag coefficient, it depends only on the Reynolds number and on object's shape, not its size. The coefficient is defined as:

$$
c_{d}=\frac{2 F_{d}}{\rho U^{2}{ }_{\text {mean }} A}
$$

Where:

$F_{d}$ is the drag force;

$\rho$ is the fluid's density;

$\mathrm{U}_{\text {mean }}$ is the mean velocity;

$\mathrm{A}$ is the projected area (product of thickness and diameter of cylinder). 
It was plotted as function of the area in front of the wall (from $x=0 \mathrm{~m}$ to $\mathrm{x}=10 \mathrm{~m}$ ) and then the area in the shadow zone of the cylinder (from $x=25 \mathrm{~m}$ to $\mathrm{x}=35 \mathrm{~m}$ ) for different values of Reynolds number.

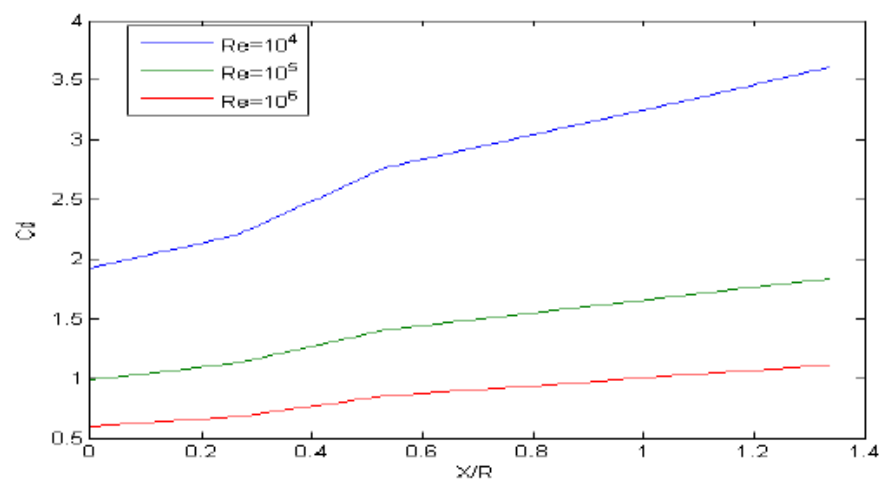

Figure 9 Drag coefficient in front of the cylinder at the center $y=17.5 \mathrm{~m}$.

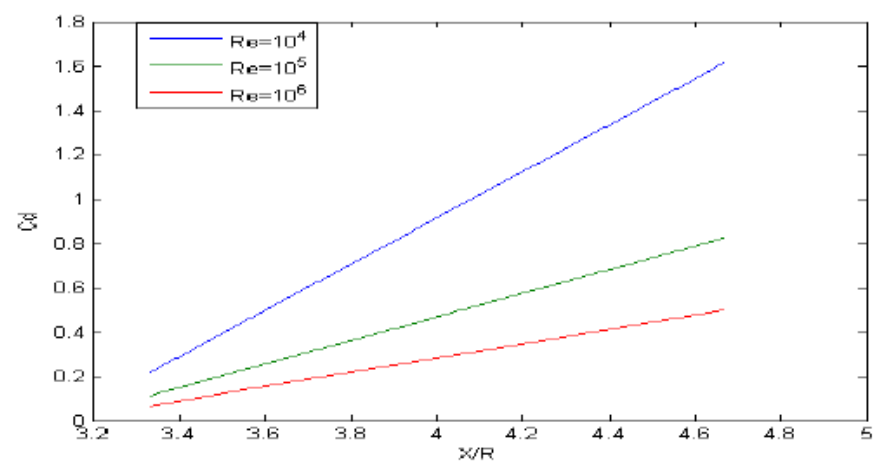

Figure 10 Drag coefficient behind the cylinder at the center $y=17.5 \mathrm{~m}$.

\section{SUMMARY}

This paper analyzed, by using CFD simulations of COMSOL Multiphysics software, the flow field concerning the complex interaction of a cylindrical structure with a fluid using the approach based on Reynolds Averaged Navier-Stokes (RANS).

Regarding velocity and pressure field simulations, it is showed an increase of pressure and velocity in the area in front of the wall from which the flow field is generated, consistent with the physical model analyzed, and a depression zone in the shadow zone of the cylinder.

As time changes, however, the pressure value increases throughout the computation domain, in according with field experiments by Boccotti $(1995,1996)$. This pressure field trend is then described into two plots, where it was showed the increase of pressure (normalized respect the maximum pressure) as function of the horizontal distance in front of the cylinder and its decrease in the shadow zone of cylinder itself.

Regarding drag coefficient, it can be noted that its trend is inversely proportional to the Reynolds number, as the theory suggests. In front of the cylinder, where the flow field is more turbulent compared to the shadow zone, for any Reynolds number, it has a value higher than the value in the shadow zone; this is coherent with the physical problem and the simulation made using COMSOL.

These simulations have required a relatively short time from a computational point of view, underlining the ability of this particular software to deal with complex problems such as those 
of a time-dependent turbulent flow. The use of numerical simulations during the design phase is useful because we can make predictions on a large number of case studies avoiding, at least in the initial phases, the realization of numerous prototypes.

It allows to perform, in a relatively simple way, and in any case, ever simpler than the real investigation, parameterizations for different initial configurations, both for geometries and for boundary conditions, allowing to evaluate the responses of the components in question to operating conditions close to physical reality.

Another great advantage of CFD is the independence with respect to the scale factor: this allows the elimination of problems, sometimes very important or of difficult and expensive resolution, of visualization of the fluid dynamic parameters in the simulation on real prototypes.

\section{REFERENCES}

[1] Barbaro, G., A new expression for the direct calculation of the maximum wave force on vertical cylinders, Ocean Engineering, vol. 34, pp. 1706-1710, 2007.

[2] Barbaro, G., Estimating design wave for offshore structures in Italian waters, Proceedings of the Institution of Civil Engineering, Maritime Engineering, vol. 164, pp. 115-125, 2011.

[3] Barbaro, G., Foti, G., Sicilia, C.L., Wave forces on upright breakwater, evaluation and case study, Disaster Advances, vol. 6, pp. 90-95, 2013.

[4] Barbaro, G., Foti, G., Sicilia, C.L., Maximum wave forces: evaluation and case studies. Proceedings of the 32nd International Conference on Ocean, Offshore and Artic Engineering OMAE (Nantes, France), 2013c.

[5] Barbaro, G., Foti, G., Sicilia, C.L., Evaluation of the horizontal wave forces on piles. Journal of Air, Soil and Water Research, vol. 7, pp. 103-110, $2014 \mathrm{~b}$.

[6] Puntorieri, P., Barbaro, G., Fiamma, V.,Experimental study of the transient flow with cavitation in a copper pipe system, International Journal of Civil Engineering and Technology 8(9), pp. 1035-1041, 2017

[7] Alsaydalani, M.O.A. A study into the motion of water in a rotating cylindrical basin using a discretization method. International Journal of Civil Engineering and Technology 10(2), pp. 1191-1205,2019

[8] Vinogradov, E.A., Nikiforov, A.V., Kochneva, A.A. Computational fluid dynamics study of ventilation flow paths on longwall panel. International Journal of Civil Engineering and Technology10(2), pp. 1140-1147,2019

[9] Muthu Subramanian, S., Manjula, R. Numerical simulation and response study of vertical cylinder under breaking waves. International Journal of Civil Engineering and Technology10(1), pp. 1781-1791,2019

[10] Boccotti P., Inertial wave loads on horizontal cylinders: a field experiment, Ocean Engineering, Vol. 23, No. 7, pp. 629--648, 1996.

[11] Boccotti P., A field experiment on the small-scale model of a gravity offshore platform, Ocean Engineering, Vol. 22, No. 6, pp. 615-627, 1995. 